\title{
Principle and implementations of a refracto-nephelo-turbidi-meter for seawater measurements
}

B. Hou1, P. Grosso1,2, J.L. de Bougrenet de la Tocnaye 1 and Marc Le Menn3

1) TELECOM Bretagne

Optics Department

Technople Iroise

29238 Brest, France

2) UMR CNRS 6082 FOTON

3) Service Hydrographique et Océanographique de la Marine (SHOM)

29228 Brest, France

E-mail: philippe.grosso@telecom-bretagne.eu

Abstract. Salinity and turbidity are two important seawater properties for the physical oceanography. The study of physical oceanography requires a compact high-resolution in-situ salino-turbidi-meter to measure these two parameters in different ocean zones. Refractometry has been proved to be an effective method to measure seawater salinity from light beam deviation. Previous studies have shown that the transmission and scattering of light in a turbid medium impact the light beam deviation measurements, which makes the combination of salinity and turbidity measurement with the same sample mandatory. In this paper, we analyze the requirements and challenges of refracto-turbidi-meter design from their measurement principles and correlations. According to these requirements, we propose a miniature refracto-nephelo-turbidi-meter with a CCD, which has a salinity measurement resolution of $2 \mathrm{mg}^{\mathrm{kg}}{ }^{-1}$ and a turbidity measurement resolution of $1 \%$ of the measurement range. Based on this refracto-nephelo-turbidi-meter, different embodiments are discussed to meet the different requirements for different ocean zones. 


\section{Introduction}

The study of physical properties of seawater requires to measure temperature, salinity and pressure with high resolutions and accuracies. In order to describe the medium, chemical and optical properties need also to be measured. Turbidity is one of them. Measurement sensors can be mounted on drifting floats as ARGO ones [1]. In this case, the development of in situ multifunctional sensors is valuable. In general, a set of these properties at different geographical positions is most valuable for the study of oceanography. This makes the research of in-situ multi-functional sensors valuable. Among these properties, attention has been focused on salinity and turbidity. In fact, temperature, salinity, and pressure are used to calculate density which variations create ocean currents. Concerning optical properties, turbidity is one of the most frequently measured quantities as it allows to quantify living and sedimentary particles concentrations and the penetration depth of light in the medium. Oceanographic studies are generally made in coastal zones or in open ocean areas. The resolution requirements for measuring salinity and turbidity are quite different in these zones. To keep a good sensitivity, transmissiometers must have a long path-length and scatterometers a high resolution. In the same way, in order to detect small density variations, salinity measurements need a high resolution. In coastal areas, suspended particles concentrations are higher and transmission path length must be smaller to avoid multiple reactions and to keep valuable the conditions of the Beer-Lambert relation. Due to the fact that coastal areas are generally characterized by high temperature and salinity gradients, resolution specifications on salinity measurements are lower. According to these requirements and application areas, it is meaningful to study the design of a high resolution in-situ salino-turbidi-meter for different scenarios.

Previous works have demonstrated an optical refractometer to measure the salinity of seawater based on laser beam deviation measurement with a 1-D Position Sensitive Device (PSD)[2]. This refractometer has been containerized and tested in real condition (2000 meters in open ocean)[3]. The resolution for measuring salinity reaches $0.002 \mathrm{~g} / \mathrm{kg}$ with a measurement range from $0 \mathrm{~g} / \mathrm{kg}$ to $40 \mathrm{~g} / \mathrm{kg}$. Further research to substitute Charge-Coupled Device (CCD) to PSD improved the resolution of nearly 1.5 times compared to PSD-based refractometer [4]. For turbidity measurement, we have analyzed the possibility of measuring salinity and turbidity simultaneously based on our refractometer from the same water sample and studied the interaction between turbidity and salinity measurements [5]. A part of this correlation is caused by the combination of transmitted and scattered light in turbid medium, which can't be separated by PSD. That is one of the reasons why PSD is inappropriate to measure the light beam deviation. Another artifact is related to the gaussian beam projection angle. When the gaussian beam projected on a plane at a non-perpendicular angle, it forms an asymmetric gaussian beam seen by the sensor as a center shift. According to these two interactions, it is mandatory to measure the turbidity for salinity measurement in a turbid medium. In this paper, we propose two new high resolution in-situ salino-turbidi-meters derived from these works. A brief summary of the salinity and turbidity measurements as well as their correlations are discussed in section 2. According to the requirements and challenges pointed out in section 2, two new salino-turbidi-meters are presented in section 3 .

A further discussion on configurations and applications of these two salino-turbidimeter is carried out in section 4 . 


\section{Requirements and Technical Challenges}

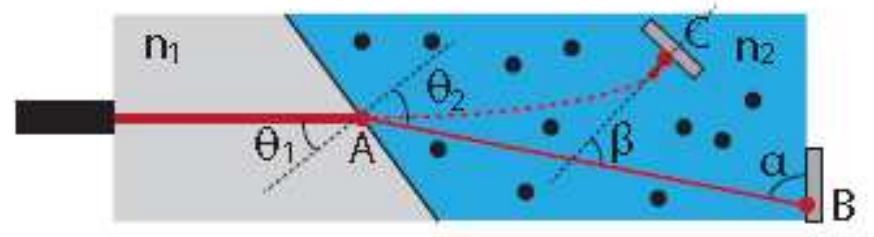

Figure 1. Principle of refractometer and the turbidity measurement

\subsection{Principle of refractometer}

UNESCO/IOC SCOR/IAPSO working group 127 (WG127)[6] has defined the measurement of seawater refractive index as one of the methods to measure the absolute salinity of seawater. Figure 1 shows the principle of a basic refractometer. A light source generates a beam of light, which propagates in the prism with a known refractive index $\mathrm{n}_{1}$. At the interface between the prism and the seawater sample, the light beam is redirected into the seawater and deviated to a light position sensor at position B, which measures the light spot position. With this light spot position, according to the Snell-Descartes law, the refractive index $\mathrm{n}_{2}$ of the seawater sample can be calculated. Our previous work included the design and implementations of a refractometers with a PSD and a V-shape refraction surface[2]. The resolution of this refractometer reached $10^{-7}$.

It should be mentioned that the refractive index measurement depends on the seawater temperature varying in the range $-2^{\circ} \mathrm{C}$ to $40^{\circ} \mathrm{C}$. The variation of temperature impacts the measurement:

Sellmeier coefficient: the variation of temperature changes the wavelength of the light source, which further impacts the refractive index of the prism.

Thermo-optical coefficient: the variation of temperature changes the refractive index of the prism.

Thermal expansion coefficient: the variation of temperature changes the dimension of the prism.

These facts require a refractometer design with a temperature compensation feature to minimize the refractive index change caused by temperature variation. Previously in our refractometer, we used two prisms, one had positive thermo-optical coefficients while another one had the negative thermo-optical coefficient, to compensate the refractive index change caused by the temperature variation. This compensation works in quasi-static conditions as in dynamic conditions because the two prisms are always at the same temperature as they are submitted to the same temperature variations and as their thermal mass is the same. The composition of glasses doesn't change significantly their density and then their weight.

A miniature design significantly reduces both temperature expansion and thermal inertia.

\subsection{Principle of turbidity measurement}

Turbidity is the cloudiness or haziness of a fluid caused by individual particle. Turbiditymeter measures transmitted or scattered light at different angles, as shown in figure 1. At point $\mathrm{C}$, a light sensor is placed at an angle $\alpha$ to the light propagation 
direction. When light propagates in the turbid water sample, the light is scattered in all the directions. The portion of light detected at an angle $\alpha$ could be used to describe the turbidity of the water sample. Among these measurement angles, two of them are the most used: $0^{\circ}$ and $90^{\circ}$. At $0^{\circ}$, transmission of light is measured a $\mathrm{s}$ shown in point $\mathrm{B}$, while scattering is measured at $90^{\circ}$ are called nephelometer. The most advanced turbidi-meters combine the nephelometer with the measurement at several different angles and combine them in a ratio of nephelometric values and a weighted sum of values from all the detection angles to assess turbidity [6]. We have verified the feasibility of measuring turbidity based on a refractometer from the transmission direction (09). The turbidity $\mathrm{T}$ can be calculated from the following equation:

$$
T=\frac{1}{l} \ln \left(\frac{I_{t r}}{I_{0}}\right)
$$

where Itr is the transmitted light intensity measured at the transmission direction, $\mathrm{I}_{0}$ is the original light source intensity and $l$ is the light path in water. From this equation, it is clear that the reference light source intensity needs to be measured and controlled with an incertainty better than $1 \%$ to calculate turbidity. According to this equation, the turbidity derivative $\mathrm{T}$, can be expressed as:

$$
T^{\prime}=\frac{d I_{t r}}{l \times I_{t r}}
$$

From (2), to improve the resolution of this method, the light path length $l$ in water can be increased, using a higher resolution light intensity sensor, or increasing the incident light intensity. We have verified this point by several simulations and experiments based on our refractometers [5]. A challenge is that the light intensity measured by the detector at $0^{\circ}$ is a combination of the transmitted light and the scattered light. Furthermore, the use of laser module as the light source causes interference by the particles in the water, which further disturbs the measurement of transmitted light intensity.

The only available information from a PSD is the gravity center and total power of the light spot so that light intensity distribution would need to be measured in order to separate the transmitted light from the mixed light. This can be made with a CCD sensor. Therefore, a CCD with a low pass band filter has been preferred to eliminate the disturbance caused by interference and separate the transmitted light as shown in the figure 2.

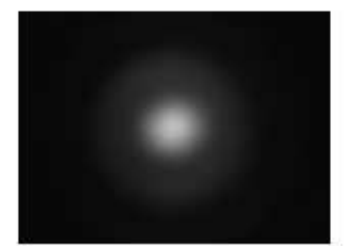

(a) originsl image for 0 NTU

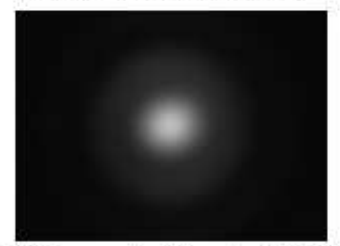

(c) image after filter for 0 NTU (d) image after filter for 444 NTU

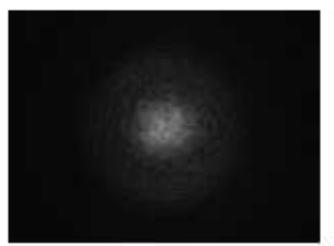

(b) orignal image for 444 NTU

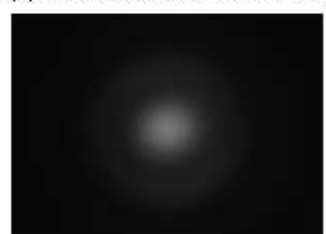

Figure 2. The captured scattered light and interference by a camera, and the corresponding images after low pass filter 


\subsection{Correlations between refractive index and turbidity measurement}

Another issue related to the combination of transmitted and scattered light is that the PSD calculates the gravity center of the incident light, which includes both the gravity center of the transmitted spot and the scattered light. This results in an error when using PSD to measure the light beam deviation in a turbid medium. Due to this reason, PSD is not appropriate for a refractometer in a turbid medium. Besides the combination of transmitted light and scattered light, other factors impact the salinity measurement in a turbid medium as well. According to the principle of refractometer illustrated in section 2.1, the refractive index is measured by the light beam deviation. However, we have found that when a Gaussian beam projected to a plane with an angle of $\alpha$ as shown in figure 1, the projection spot is still a Gaussian spot with the same spot size but with a center shift. The shift distance is proportional to the turbidity of the medium, the squared spot size and inversely proportional to the tangent of the angle $\alpha$. We established in [5] the following relation:

$$
I=I_{0} e^{\frac{-\left(x-x_{p}\right)^{2}}{2 \sigma^{2}}} e^{\frac{-T\left(x-x_{p}\right)}{u}-T l}=e^{\frac{-\left(x-x_{p}+\frac{T \sigma^{2}}{u}\right)^{2}}{2 \sigma^{2}}} I_{0} e^{\frac{T^{2} \sigma^{2}}{2 u^{2}}-T l}
$$

In which $I_{0} e^{\frac{-\left(x-x_{p}\right)^{2}}{2 \sigma^{2}}}$ is a standard gaussian beam, $T$ is the attenuation coefficient, $l$ is the light path of the center, $u$ is the coefficient that describes the different attenuation inside the beam, $x_{p}$ is the original peak position and $\sigma$ is the laser beam size. From (3), it is easy to conclude that the new laser spot is still a gaussian spot but with a peak shift of :

$$
-\frac{T \sigma^{2}}{u}
$$

Because of this new gaussian center variation law, both refractive index and turbidity contribute to the light beam deviation, which results in an error to measure the refractive index from the light beam deviation. Based on this correlation, it is necessary to combine the turbidity and salinity measurements with the same seawater sample and the turbidity need to be calculated first in order to accurately indicate the salinity. Furthermore, when the gaussian beam is not collimated, the projection spot with a non-perpendicular angle becomes asymmetrical, which impacts the gravity-center-based spot location method, such as PSD and centroid algorithm. A new method, which tracks the location that divides the mass of the spot into two equal parts, introduced in our previous study [5] has been proved to be a good solution to this problem. Another correlation between the salinity and turbidity measurements is that the suspended particles have different refractive index from the seawater. A part of light refracted by the particles is captured by the position sensor as well, which impacts the light beam deviation. However, it should be mentioned that the light beam deviation caused by the particle-refracted light is much smaller than the required beam deviation resolution so that it is not a key issue for the refractive index measurement. Besides the impact to salinity measurement caused by turbidity, the configuration of refractometer affects the turbidity measurement as 
well. As shown in equation (1), the attenuation of the transmitted light is related to the geometrical light path length $l$ in water. However, the light path length changes according to different refractive indices of seawater samples. For two different seawater samples with same turbidity, but different refractive index, the measured light intensities at $0^{\circ}$ are different due to different light path lengths. That is to say, the design of the refracto-turbidi-meter needs to minimize the light path length variation caused by the refractive index changes.

\subsection{Refracto-turbidi-meter requirements}

According to the principle of salinity and turbidity measurement methods as well as the correlation between them, it is necessary to combine these two measurements with the same seawater samples. This results in some requirements to guide the design of refracto-turbidi-meter.

(i) Reduce refractive index variation caused by the temperature change

(ii) Minimize the sensor size to reduce the thermal expansion

(iii) Maximize the light path length in water to improve the turbidity resolution

(iv) Measure the original light intensity as the reference to calculate turbidity

(v) Select different light source types according to the particle size in order to avoid interference

(vi) Use CCD to eliminate the disturbance caused by the combination of interference and scattered light

(vii) Use ratio nephelometer to improve the turbidity resolution

(viii) Keep the beam projected on the position sensor at an angle of $90^{\circ}$ in order to avoid the beam deviation caused by turbidity

(ix) Minimize the light path length variation caused by refractive index change.

Therefore, any design of refracto-turbidi-meter should take the considerations of these requirements.

\section{Design and Implementation}

According to the requirements and challenges discussed in last section, a new miniature high resolution refracto-turbidi-meter is designed. Figure 3 depicts the new design of a refracto-turbidi-meter. This new model only has one piece of prism. The light beam first enters the prism from the left corner and reaches the first refraction surface AO.

It is then refracted into the medium and refracted again at the refraction surface $\mathrm{BO}$. This refraction directs the beam to a mirror CD, which redirects the upwards beam to the right side of the prism, where it meets the second mirror EF and redirects to a 20 $\mathrm{mm}$ long groove at the right side of the prism. Finally, the beam propagates through the groove and reaches the right bottom of the prism, where a CCD is used to measure the beam deviation caused by the water refractive index variation. At the inner surface of the groove, a surface treatment is used to absorb the reflected light to avoid the influence of the scattering inside the groove. 


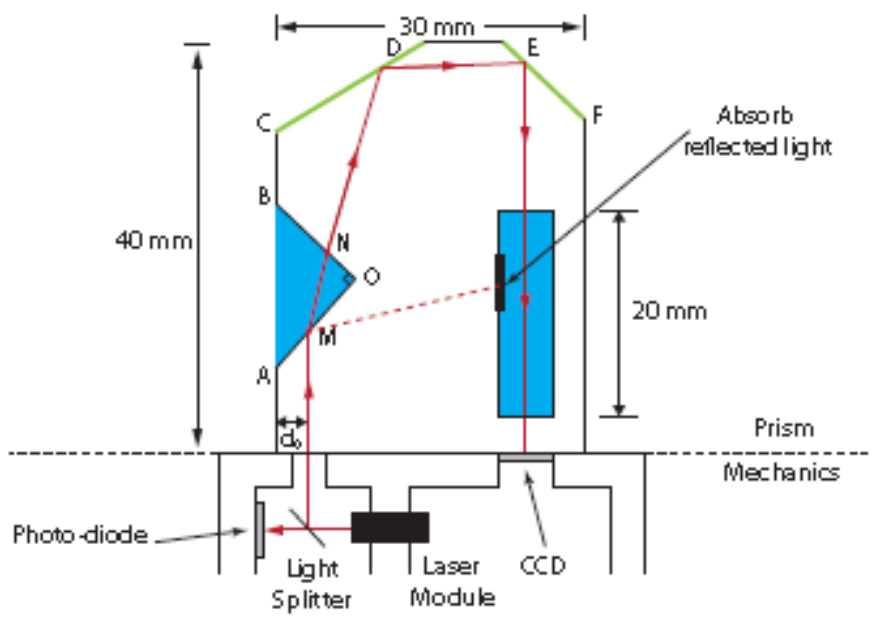

Figure 3. Prototype of miniature refracto-turbidi-meter

\subsection{Refractive index measurement}

As discussed in section 2.1, temperature has a strong impact on the in-situ refractive index measurement. In our design, we use a single prism to compensate the refractive index change caused by the temperature variation. The refractive index is not only associated to temperature but also to wavelength, illustrated by Sellmeier equation [8]:

$$
n^{2}(\lambda(T))=1+\frac{B_{1} \lambda(T)^{2}}{\lambda(T)^{2}-C_{1}}+\frac{B_{2} \lambda(T)^{2}}{\lambda(T)^{2}-C_{2}}+\frac{B_{3} \lambda(T)^{2}}{\lambda(T)^{2}-C_{3}}
$$

When the temperature increases, the wavelength of the laser as well, which further results in the decrease in glass refractive index, according to (5). The variation caused by the wavelength change $\Delta \mathrm{n} \lambda<0$ (when $\Delta \mathrm{T}>0$ ) can be used to compensate the refractive index variation induced by temperature change $\Delta \mathrm{nT}$ (when $\Delta T<0)$. To choose a glass with this feature, we plotted two functions, $d n / d T=f_{1}(T)$ and $d n / d \lambda=f_{2}(T)$, for several Schott glasses at a wavelength of $635 \mathrm{~nm}$ in $20{ }^{\circ} \mathrm{C}$, and found that the glass $\mathrm{N}-\mathrm{ZK} 7$ best fits our requirements. Figure 4 plots the two functions and the refractive index variation from $0_{-} \mathrm{C}$ and $40 \_\mathrm{C}$ for $\mathrm{N}-\mathrm{ZK} 7$. As shown in figure 4 , the refractive index variation of $\mathrm{N}-\mathrm{ZK} 7$ from $0{ }^{\circ} \mathrm{C}$ and $40{ }^{\circ} \mathrm{C}$ is only $7.5 \mathrm{x}$ $10^{-7}$.

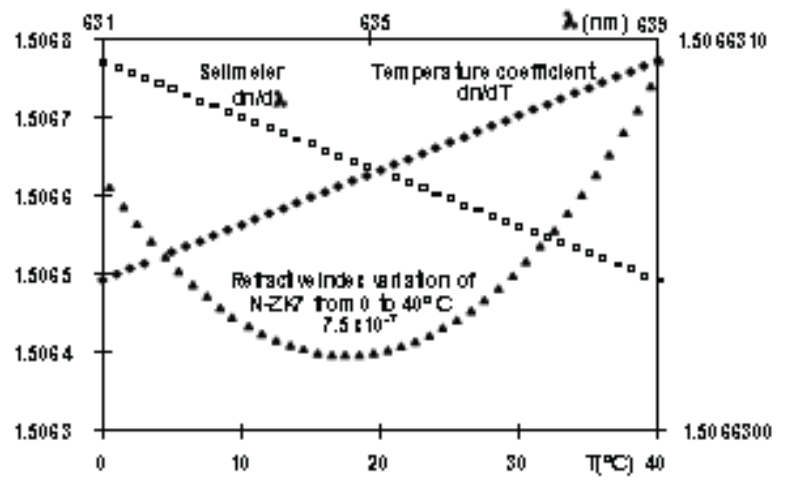

Figure 4. The temperature coefficient and Sellmeier coefficient of glass N-ZK7 when temperature varies from $0{ }^{\circ} \mathrm{C}$ to $40^{\circ} \mathrm{C}$ 
Furthermore, this new design has a very small size prism (40 mm×30 mmx20 mm), resulting in a very small thermal expansion. The refractive index measurement resolution of this design can be evaluated by using the geometric method and differential method to build the relationship between the spot position and the refractive index. According to the calculation results, the relationship between the spot position $\mathrm{P}$ and seawater refractive index $\mathrm{n}_{\mathrm{e}}$ can be approximately obtained by a curve polynomial fitting. The equation of the fitting curve of the second order is shown in equation 4 , with a standard deviation of $4.86 \times 10^{-7}$.

$$
n_{e}=0.000032 P^{2}+0.006 P+1.34
$$

When the refractive index changes from 1.330 to 1.350 , the spot moves a distance of about $2.2 \mathrm{~mm}$ from $-1.1 \mathrm{~mm}$ to $+1.1 \mathrm{~mm}$. According to the spot location resolution (better than $0.1 \mu \mathrm{m}$ ) checked in our previous work by using a CCD [5], the resolution of measuring seawater refractive index reaches $0.9 \times 10^{-6}(\approx 1 \mathrm{ppm})$ which is equivalent to the absolute salinity of $2 \mathrm{mg} \mathrm{kg}^{-1}$. Another issue related to the refractive index measurement is the Gaussian spot center shift according to the projection

angle (inversely proportional to the tangent of projection angle). To minimize this center shift, the light beam enters the groove at an angle of $90^{\circ} \pm 0.865^{\circ}$ with the range of refractive index from 1.33 to 1.35 .

\subsection{Turbidity measurement}

The turbidity measurement uses the measurement in the transmission direction. A CCD is used to capture the transmitted light intensity. As discussed in section 2.2, the transmitted light is mixed with the scattered light and interference, a CCD with a low pass filter can eliminate the scattered light and interferences. The turbidity resolution based on the transmitted light measurement is inversely proportional to the light path length in water. In our previous work, we have obtained a resolution of 1:15 NTU (Nephelometric Turbidity Units) in the range from 0 to 20 NTU. The corresponding light path length in water is about $6.35 \mathrm{~mm}$. The turbidity measurement resolution of this new design can be calculated as: $R_{t}=1.15 \times 6.35 /(20$ $+\mathrm{MN}$ ), in which $\mathrm{MN}$ is the length of light path between the two refraction surfaces. This distance is directly affected by the incident light position do as shown in Fig.3. The light path distance between two refraction surfaces decreases from $11 \mathrm{~mm}$ to 1.8 $\mathrm{mm}$, when the incident beam position moves from $3 \mathrm{~mm}$ to $8 \mathrm{~mm}$. If we use $5 \mathrm{~mm}$ as the incident beam position do, the expected turbidity measurement resolution would be $R_{t}=1.15 \times 6.35 /(20+\mathrm{MN})=0.27 \mathrm{NTU}$ in the range from 0 NTU to $20 \mathrm{NTU}$. This design also reduces the impacts caused by the light path difference with different seawater refractive indices. The variation of the light path length between two refraction surfaces is about $0.054 \mathrm{~mm}$, which is only $0.23 \%$ of the entire light path, so that it can be ignored when calculating turbidity. Another improvement is the set-up of light source. In our previous refractometers, light source was directly in contact with to the prism, making it difficult to measure the reference light intensity lo. In the new model, light source is installed with the mechanics, a light splitter is used to separate the beam into two parts: one enters into the prism for the measurement, another is measured by a photo-diode as the reference light intensity lo. This design makes the use of different light sources other than laser possible. The laser module shown in 
Fig. 3 can be replaced by a LED at wavelength $860 \mathrm{~nm}$, which is not absorbed by the natural particles and reduces interference. Another option is $530 \mathrm{~nm}$, which is used for the calibration of submarine visibility model and has less absorption by the seawater. Besides these advantages brought by the light source set-up, the separation of the light source and the prism is a mandatory configuration for the temperature compensation. As mentioned in section 3.1, the refractive index variation caused by the temperature change is compensated by the positive temperature coefficient variation and negative Sellmeier coefficient variation with the same temperature change, which requires that the light source and measured sample keep the same temperature. This separation enables a good thermal isolation to avoid the different temperatures, caused by the light source heating, between the light source and the prism. The attenuation based turbidity measurement is not sensitive in ultra low and ultra high turbid medium. To improve the performance of the refracto-turbidi-meter, a new photo-diode is introduced to measure the scattered light at $90^{\circ}$ as shown in figure 5. This new refracto-nepheloturbidi-meter equips with a mirror at $45^{\circ}$ to the light propagation direction at the right side of the groove, which reflects the diffused light to the photo-diode B placed inside the mechanics. With this configuration, the turbidity Tu is calculated by the photodiode B (/90) and the light intensity obtained by the CCD (Iccd) as:

$$
T u=\frac{I_{90}}{\alpha_{1} I_{90}+\alpha_{2} I_{C C D}}
$$

In which $\alpha_{1}$ and $\alpha_{2}$ are two constant coefficients. It should be noticed that the reference light intensity lref given by the photo-diode $A$ is not used in the calculation. The reason is that equation (7) is a ratio between the $90^{\circ}$ scattered light and the weighted sum of transmitted light and scattered light, which is independent of the incident light intensity. However, both the nephelometer standard ISO 7027[9] and USEPA Method 180.1[10] only have a measurement range from 0 to 40 NTU. The photo-diode $B$ introduced in this design is used to improve the performance in ultra low turbid medium, for the moderate turbidity measurement, the attenuation based measurement can be used. Based on this reason, we keep the photo-diode A to measure the reference intensity for the attenuation based turbidity measurement.

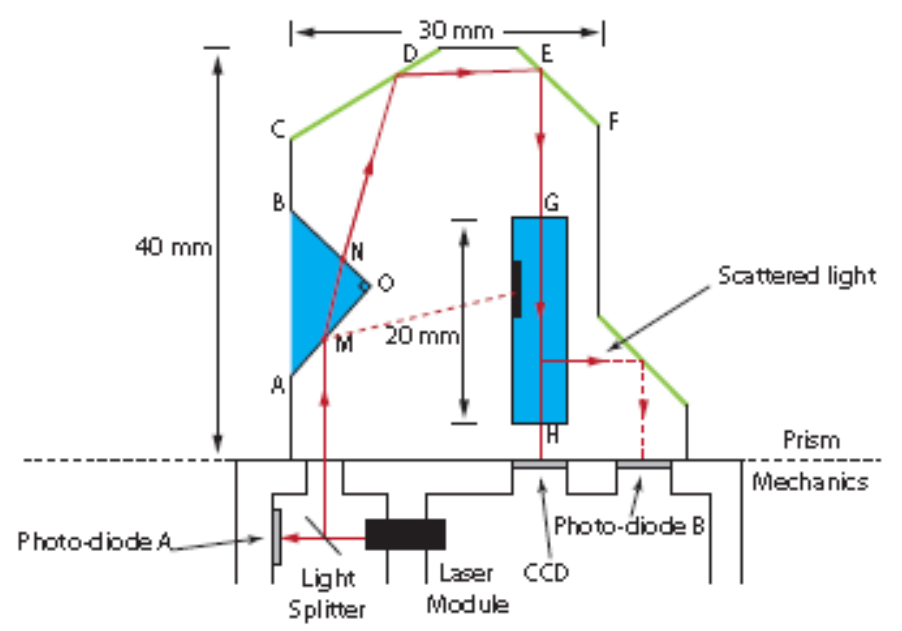

Figure 5. The prototype of miniature refracto-nephelo-turbidi-meter

This turbidi-meter is expected to have the same resolution as the one proposed in last section ( $1 \%$ of the measurement range), but owns better performance in ultra low turbidity medium. 


\section{Device Customization}

As we have discussed in the introduction section, the measurement in different zones of the ocean has different requirements. Accordingly, the salino-turbidimeterintroduced in this paper should have different configurations to fulfill the different application scenarios. In open ocean, the seawater is clear enough so that it is not necessary to measure turbidity, and there is no impact to the salinity measurement caused by the turbidity, such as Gaussian center shift. Thus, a configuration without the groove can be used to measure salinity in open ocean. A PSD or a CCD, can be used as the light position detection device, because there is no influence caused by turbidity. In offshore area, the turbidity stays in a low level

(usually smaller than $20 \mathrm{NTU}$ ), the refracto-turbidi-meter with the $45^{\circ}$ mirror design can be used. The ratio calculated by (5) can help the turbidity measurement obtain a resolution of $1 \%$ of the measurement range. Boss and W. Scott Pegau have pointed out that $117^{\circ}$ is a better turbidity detection angle for the ocean turbidity measurement [11], it is possible to change the angle of the $45^{\circ}$ mirror to $31.5^{\circ}$ in order to reflect the scattered light at $117^{\circ}$. As the turbidity impacts the refractive index measurement, using a CCD as the light position detection device is mandatory for the measurement of light beam deviation. Different from the offshore and open ocean cases, the coastal areas have a high turbidity, which might be out of the nephelometer measurement range (usually $40 \mathrm{NTU}$ ). The CCD based transmissometer can be used to measure the turbidity in the high turbidity case. Furthermore, the use of CCD combined with the advanced post-processing algorithms, the influence to salinity measurement caused by turbidity can be reduced.

\section{Conclusion}

The study of physical oceanography requires the in-situ measurement of salinity and turbidity with the same seawater sample. The refractometer based salinity measurement requires that the sensor has smaller thermal expansion and less temperature influence. To obtain a higher resolution turbidity measurement, the original light intensity must be measured as the reference and the sensor should contain a long light path length in water. The turbidity impacts the combination of transmitted light and scattered light at the light propagation direction. This added to the light interference caused by the suspended particles, when using a laser, make PSD inappropriate in a turbidity medium to measure the salinity. Furthermore, the Gaussian beam will have a center shift in a turbid medium when the beam projects to a plane at a non-perpendicular angle. This proves that the turbidity disturbs the salinity measurements. According to these requirements and challenges, we have designed a new refracto-turbidi-meter equipped with a CCD to reduce the turbiditycaused impacts to the salinity measurements. To improve the turbidity measurement performance in low turbid medium a refracto-nephelo-turbidi-meter is designed as well. According to the measurement principle and previous work we designed, the new refracto-turbidi-meter with different configurations can work in different application scenarios, including open ocean, offshore, and coastal areas. The expected salinity measurement resolution reaches $2 \mathrm{mg} \mathrm{kg}^{-1}$ and the expected turbidity measurement resolution is $1 \%$ of the measurement range in both the low turbid medium and the high turbid one. 


\section{References}

[1] R.H. Stewart. Introduction to Physical Oceanography. University Press of Florida, 2009.

[2] D Malarde, Z Y Wu, P Grosso, J-L de Bougrenet de la Tocnaye, and M Le Menn. High-resolution and compact refractormeter for salinity measurements. Measurement Science and Technology, No 1(20), 2009.

[3] M Le Menn, J L de Bougrenet de la Tocnaye, P Grosso, L Delauney, C Podeur, P Brault, and O Guillerme. Advances in measuring ocean salinity with an optical sensor. Measurement Science and Technology, 22(11):115202, 2011.

[4] Bo Hou, Zong Yan Wu, Jean-Louis de Bougrenet de la Tocnaye, and Philippe Grosso. Chargecoupled devices combined with centroid algorithm for laser beam deviation measurements compared to a position-sensitive device. Optical Engineering, 50(3):033603, 2011.

[5] B. Hou, P. Grosso, Z.Y. Wu, and J.L. de Bougrenet de la Tocnaye. A turbidi-meter based on a refractometer using a charge-coupled device. Optical Engineering, 51(02), 2012.

[6] IOC, SCOR, and IAPSO. The international thermodynamic equation of seawater 2010:

Calculation and use of thermodynamic properties. 2010.

[7] Mike Sadar. Turbidity measurement: A simple, effective indicator of water quality change, 2011.

[8] G. Ghosh. Handbook of thermo-optic coefficients of optical materials with applications. Handbook of Optical Constants of Solids. Academic Press, 1998.

[9] Iso7027: Water quality - determination of turbidity. International Standard, 1999.

[10] APHA, AWWA, and WPCF. Standard methods for the examination of water and wastewater. pages 2-12, 1989.

[11] Emmanuel Boss and W. Scott Pegau. Relationship of light scattering at an angle in the backward direction to the backscattering coefficient. Appl. Opt., 40(30):5503-5507, Oct 2001. 